\title{
TRANSCERVICAL, PARATRACHEAL RESECTION OF A POSTERIOR MEDIASTINAL MASS
}

\author{
Tetsuo Kido, MD, Hiroyuki Nishi, MD, Masaaki Nakahara, MD, and Kazuyasu Nakao, MD, Osaka, Japan
}

Standard thoracotomy and video-assisted thoracic surgery (VATS) are treatment methods for benign upper mediastinal neurogenic tumor, depending on the site and size. The supraclavicular approach ${ }^{1}$ is considered suitable because it can be performed by avoidance of a thoracotomy; however, postoperative nerve injury may occur. We obtained satisfactory results in a patient using the paratracheal approach parallel to the thyroid gland, a new minimally invasive approach, for upper mediastinal tumor resection.

Clinical summary. The patient was a 58-year-old man who had an abnormal shadow on the chest $\mathrm{x}$-ray film 12 years earlier. The shadow was ignored because he was otherwise free of symptoms. He sought medical assistance in March 1998, because slight enlargement of the abnormal shadow was found. On chest radiography a tumor was detected in the right upper thoracic space. Thoracic computed tomography revealed a large oblong homogeneous mass $4 \times 3 \mathrm{~cm}$ in size in contact with the first and second right thoracic vertebrae. The tumor was diagnosed on imaging as a neurogenic tumor.

In June 1998 surgery was performed with the use of general anesthesia with the patient in the supine position. After a 7$\mathrm{cm}$ right cervical incision had been made, the platysma and the right anterior cervical muscles were cut to expose the inferior pole of the right thyroid gland. The sternocleidomastoid muscle and the right common carotid artery were then dissected out and retracted. Cautery was avoided, and taping of the nerve was not performed. After exposure and identification of the right inferior thyroid artery and the right recurrent laryngeal nerve, the right inferior thyroid artery was ligated and dissected. A paratracheal approach between the thyroid gland and the common carotid artery, through the thoracic vertebral space to the tumor, was used (Fig 1). Dissection was initiated at the upper margin of the tumor, which was confirmed as having arisen from the sympathetic trunk. The right vertebral artery was identified, and dissection proceeded to the lower margin, with the artery placed externally. The junction of the lower margin of the tumor and the sympathetic trunk was identified, and the lower margin of the tumor was dissected out. The junction between the upper margin of the tumor and the sympathetic trunk was freed and

From the Department of Surgery, Osaka Police Hospital, Osaka, Japan.

Received for publication Oct 2, 1998; accepted for publication Dec 2, 1998.

Address for reprints: Tetsuo Kido, MD, Department of Surgery, Osaka Police Hospital, 10-31, Kitayama-chou, Tennouji-ku, Osaka, 543-8502, Japan.

J Thorac Cardiovasc Surg 1999;117:1036-7

Copyright (C) 1999 by Mosby, Inc.

$0022-5223 / 99 \$ 8.00+0 \quad \mathbf{1 2 / 5 4 / 9 6 2 1 2}$

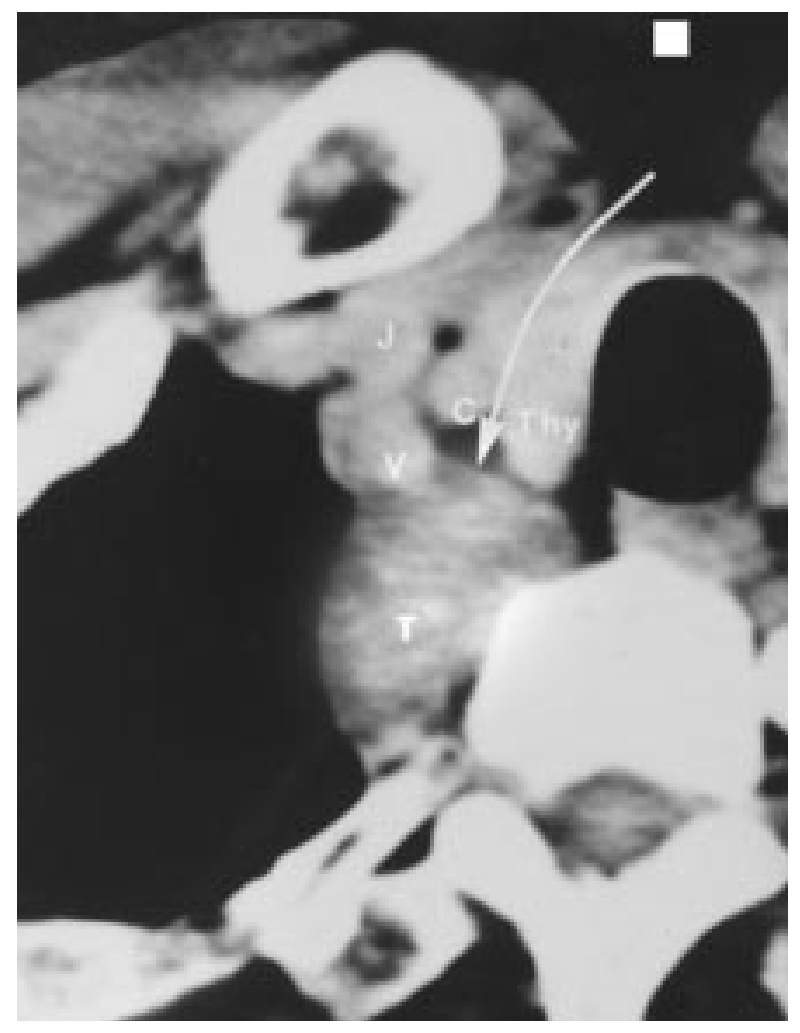

Fig 1. Paratracheal route (white arrow) along the thyroid gland to the tumor $(T)$. Thy, Thyroid gland; $C$, common carotid artery; $J$, internal jugular vein; $V$, Vertebral artery.

the tumor was then totally extracted (Fig 2). The size of the resected tumor was $4.5 \times 2.5 \mathrm{~cm}$. It was identified as ganglioneuroma on histopathologic examination. The patient had a satisfactory postoperative clinical course, and he was discharged 1 day later.

Comment. Tumors in the posterior mediastinum are often of benign neurogenic origin, and therefore a minimally invasive surgical approach is desirable. Recently, VATS has become a standard method for the resection of mediastinal tumors. ${ }^{2}$ However, use of VATS to resect upper mediastinal tumors is controversial because many vessels and nerves are present in the surrounding structures, in particular in the upper thoracic space, and operation under direct vision is thought to be safer. A supraclavicular approach ${ }^{1}$ for the resection of upper mediastinal tumors is anatomically the most direct approach to the tumor. With this approach, the nerves and blood vessels can be identified under direct vision. This method is both minimally invasive and safe. Ohara and asso- 


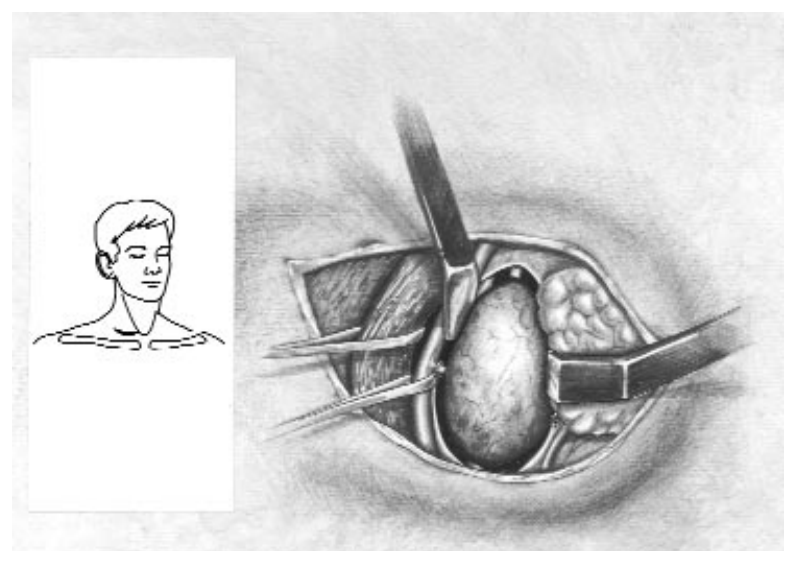

Fig 2. The extirpated tumor through the space between the thyroid and the external common carotid artery.

ciates $^{3}$ reported 4 cases of mediastinal tumor resection using the supraclavicular approach and emphasized the importance of this method. However, they also described phrenic nerve paralysis in 3 cases. In their cases, stretch trauma ${ }^{4}$ resulting from tension during taping was considered to be the main cause of phrenic nerve paralysis. To reduce the danger of phrenic nerve damage, an approach that avoids the phrenic nerve is necessary. In our case, the phrenic nerve was not crossed because the scalenus muscle was not removed. Fur- thermore, the approach to the tumor was relatively simple, inasmuch as only taping of the common carotid artery and the sternocleidomastoid muscle and external traction were required. If the tumor is of neurogenic origin with a diameter of $4.5 \mathrm{~cm}$ or less in the upper thoracic space, resection may be possible. However, if the tumor extends into the region below the third thoracic vertebra or into the entire area of the upper thoracic space, a thoracic approach may be necessary in addition to the supraclavicular approach, as in our case.

Although suitability of this approach is limited, surgical invasion is low because the muscle incision is minimized. Since a thoracotomy is not required, other advantages exist such as no lung damage. Thus we consider our approach useful and not associated with nerve damage.

\section{REFERENCES}

1. Sanders RJ, Raymer S. The supraclavicular approach to scalenectomy and first rib resection: description of technique. J Vasc Surg 1985;5:751-6.

2. Landreneau RJ, Mack MJ, Hazelrigg SR, Naunheim KS, Keenan RJ, Ferson PF. The role of video-assisted thoracic surgery in thoracic oncological practice. Cancer Invest 1995;13:526-39.

3. Ohara T, Hata E, Take A, Kamisawa O, Fukushima K, Kotoda K, et al. Supra-clavicular approach for tumors located in the superior mediastinum. J Jpn Assoc Thorac Surg 1987;49:49-53.

4. Iverson LIG, Mittal A, Dugan DJ, Samson PC. Injuries to the phrenic nerve resulting in diaphragmatic paralysis with special reference to stretch trauma. Am J Surg 1976;132:263-9.

\section{Authoritative}

The Journal of Thoracic and Cardiovascular Surgery is the most frequently cited thoracic/cardiovascular surgery journal in the Science Citation Index. An article in JTCVS is sited on average almost twice as often as those in the closest cardiothoracic journal. 